\title{
Staying connected during the COVID-19 pandemic: highlights from the Paediatric and Congenital Interventional Cardiology Early-Career Society (PICES) 2020 activities
}

\author{
Sebastian Góreczny ${ }^{1}$, Wendy Whiteside ${ }^{2}$, Tacy Downing ${ }^{3}$, Varun Aggarwal ${ }^{4}$, Gurumurthy Hiremath ${ }^{4}$, \\ Ryan Callahan ${ }^{5}$, Brian Boe ${ }^{6}$ \\ ${ }^{1}$ Department of Paediatric Cardiology, University Children's Hospital, Faculty of Medicine, Jagiellonian University Medical College, Krakow, \\ Poland \\ 2Division of Pediatric Cardiology, University of Michigan C.S. Mott Children's Hospital, Ann Arbor, MI, USA \\ ${ }^{3}$ Children's National Hospital and the George Washington University School of Medicine, Washington, DC, USA \\ ${ }^{4}$ Division of Pediatric Cardiology, University of Minnesota, Masonic Children's Hospital, Minneapolis, MN, USA \\ ${ }^{5}$ Department of Cardiology, Boston Children's Hospital and Harvard Medical School, Boston, MA, USA \\ ${ }^{6}$ The Heart Center, Nationwide Children's Hospital, Columbus, OH, USA
}

Adv Interv Cardiol 2021; 17, 1 (63): 1-5 DOI: https://doi.org/10.5114/aic.2021.104762

The Paediatric and congenital Interventional Cardiology Early-Career Society (PICES) was conceived in a hallway conversation during the 2011 Society for Cardiovascular Angiography and Interventions (SCAI) meeting in Baltimore, MD and was subsequently formally established in July of 2011 [1]. Since its inception, the organization has focused on promoting clinical education, fostering multicentre research collaboration, developing leadership skills, and promoting professional networking among the early-career (within 10 years of their training) paediatric/congenital interventional cardiologists as well as fellows-in-training. Membership is available free of charge to anyone in congenital cardiology training, congenital interventional cardiology training, or an interventional cardiology faculty early within their career. PICES is a special interest group of the SCAI, and multiple previous and current PICES executive committee members have served on the Congenital Heart Disease (CHD) council of the SCAI. Currently the society consists of more than 200 members with approximately $60 \%$ United States and $40 \%$ international representation.

The PICES executive committee through September 2020 was composed of President - Ryan Callahan, MD (Boston Children's Hospital), Vice President (Research) - Gurumurthy Hiremath, MD (University of Minnesota
Children's Hospital), Vice President (Clinical) - Wendy Whiteside, MD (C.S. Mott Children's Hospital), Secretary - Tacy Downing, MD (Children's National Hospital), and International Delegate - Sebastian Góreczny, MD, PhD (Jagiellonian University Children's Hospital). In the summer of 2020, Brian Boe, MD (Nationwide Children's Hospital) was elected the new President and Varun Aggarwal, MD (University of Minnesota Masonic Children's Hospital), the new Vice President (Research).

The virulence of COVID-19 has led to many changes in health care. In addition to the precautions and protocols adopted by hospitals and practices, professional meetings were cancelled or transitioned to virtual platforms [2]. In the world of congenital interventional cardiology, these in-person meetings allow the sharing of knowledge and networking among practitioners. One of the groups who were most severely impacted by the lack of traditional meetings were the early-career interventional cardiologists who do not have the broad experience and professional network shared by their more senior colleagues. Over the past year, PICES has made significant adjustments to maintain contact and support its members.

The main source of communication among PICES members is via an email listserv where members post 
clinical questions, collaborate on research, and share interesting topics. Similarly to previous years, the 2020 PICES listserv posted several academic activities, presentation opportunities and job postings [3].

This past spring marked the completion of the $2^{\text {nd }}$ class of the PICES Mentorship Program championed by Matthew Crystal, MD (Morgan Stanley Children's Hospital) and currently administered by our secretary Tacy Downing, MD. This grant-supported program matches young congenital interventionalists with a senior mentor. Each mentee/mentor pair connects twice monthly and exchanges site visits during the 2-year program. Throughout the program, the mentors foster the careers of the junior interventionalists by cultivating educational, research, and leadership skills opportunities. With the completion of the most recent class, a total of 6 pairs have completed the program.

One of the goals of PICES is to foster academic and clinical advancement of early career congenital interventional cardiologists around the globe through collaborative research. PICES members have been very active and shared their research and academic experiences amongst each other. Many of these projects have culminated in successful presentations at various national and international conferences. To date, PICES has completed 8 multicentre research projects with successful manuscripts [4-11]. These projects have included a total of 50+ contributing authors across 30+ different institutions (including many international centres). The most recent project evaluated the use of balloon expandable stent implantation for the treatment of transverse arch nar- rowing [12]. Currently, there are 5 active PICES research projects, ranging from variation in Fontan management to the use of drug-eluting stents in the congenital cardiac catheterization laboratory:

- Branch pulmonary artery stent and right ventricle size study - Petch Suntharos, MD (Nicklaus Children's Hospital),

- Fontan liver practice variation study - Kiran Mallula, MD (Children's Hospital of New Orleans),

- Left atrial decompression in hypoplastic left heart syndrome - Konstantin Averin, MD (Stollery Children's Hospital),

- Afterload from pressure-volume loops in coarctation stent patients - Gurumurthy Hiremath, MD (University of Minnesota Children's Hospital),

- Drug-eluting stents and anticoagulation practice pattern in congenital heart disease - Varun Aggarwal, MD (University of Minnesota Children's Hospital).

In addition, there are 2 more proposals for projects which are currently undergoing the initial planning phase:

- Pulmonary atresia with right ventricular dependent coronary circulation, different treatment options and outcomes - Daniel McLennan, MD (Children's Hospital of Wisconsin),

- Transcatheter Mitral Valve Replacement in Congenital Heart Disease - Allen Ligon, MD (Joe DiMaggio Children's Hospital).

In an effort to keep everyone abreast of all the research initiatives by PICES a project was created on ResearchGate this past year (https://www.researchgate.

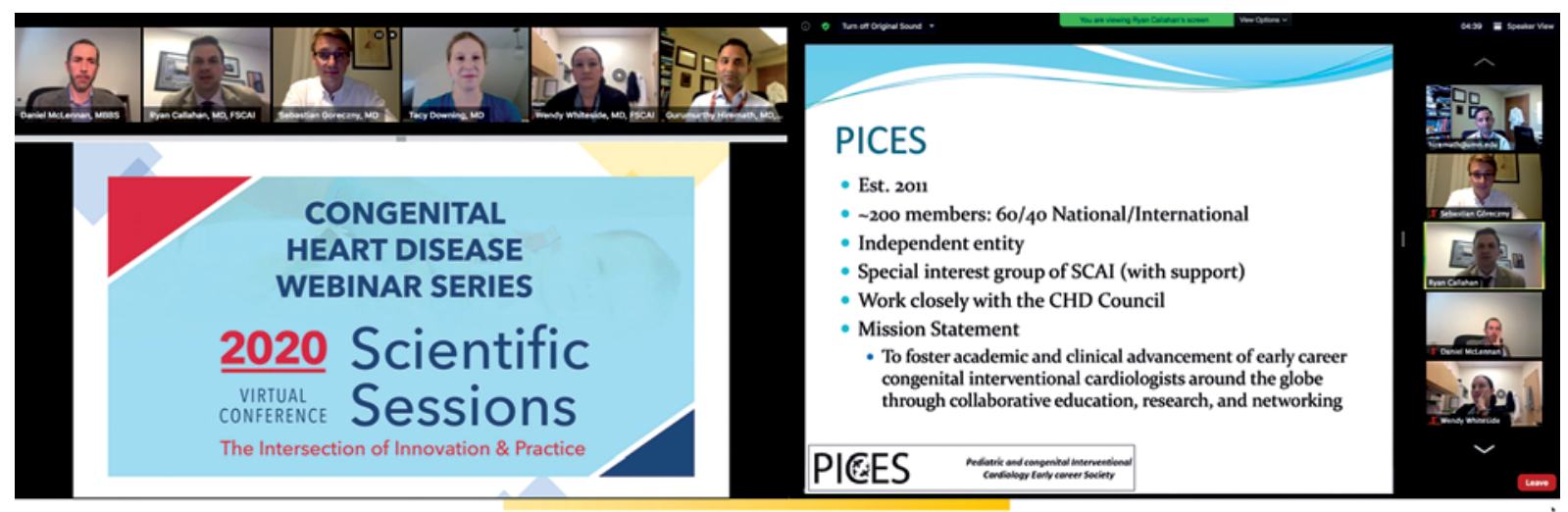

\section{Just When I Thought I} Covered All the Bases

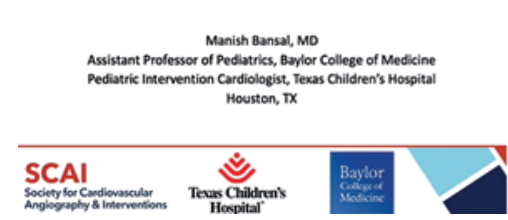

Stent Enlargement of HeartWare Outflow Cannula: How VAD Could it Be? PICES at SCAI May 2020 Hannah Fraint, M.D. Blalock-Taussig-Thomas Pediatric and Congenital Heart Center Blalock-Taussig-Thomas Pediatric
Johns Hopkins Children's Center
JOHNS HOPKINS

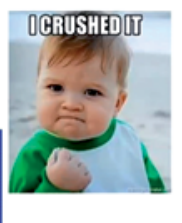

When you think you have crushed it, and then realise you have!!

Figure 1. The Paediatric and Congenital Interventional Cardiology Early-career Society (PICES) session at the Society for Cardiovascular Angiography and Interventions (SCAI) meeting 
net/project/PICES-Pediatric-and-congenital-Interventional-Cardiology-Early-career-Society). It collects all previously published PICES collaborative projects as well as updates on currently open studies.

As with most professional organizations, our members meet regularly at professional meetings. PICES had to adjust to the transition of virtual conferences due to the COVID-19 pandemic. Since our communication relies heavily on an online listserv, the PICES members were able to easily transition to a virtual format.

\section{PICES@SCAI}

SCAI Scientific Sessions 2020 were held virtually, and PICES was excited to continue to participate in this meeting on 15 June 2020 (Figure 1). President Ryan Callahan, MD gave an update on PICES during the Congenital Heart Disease Community Forum. PICES hosted an evening webinar titled "Planning Complicated Interventions and Dealing With Unpredictable Complications: How to Expect the Unexpected", with the goal of providing insight on how junior interventionalists prepare for complex cases and strategies for managing unanticipated situations. The webinar included 3 case presentations by PICES members:

- "Just When I Thought I Covered All the Bases" by Manish Bansal, MD (Texas Children's Hospital).

- "Stent Enlargement of HeartWare Outflow Cannula How VAD Could It Be?" by Hannah Fraint, MD (Johns Hopkins Hospital).

- "When You Think You Have Crushed It, and Then Realize you Have!” by Daniel McLennan, MBBS (University of lowa Stead Family Children's Hospital).

All 3 presentations were excellent, and they provided thoughtful discussions among its members during the new virtual format. Overall, the PICES session was well attended and as of March 2021, the session is available for viewing via the SCAI website (https://virtual2020.scai.org/).

\section{PICES@PICS-AICS}

The Paediatric and Adult Interventional Cardiac Symposium (PICS-AICS) also transitioned to a virtual format in 2020 (Figure 2). The PICES breakout session (12 September

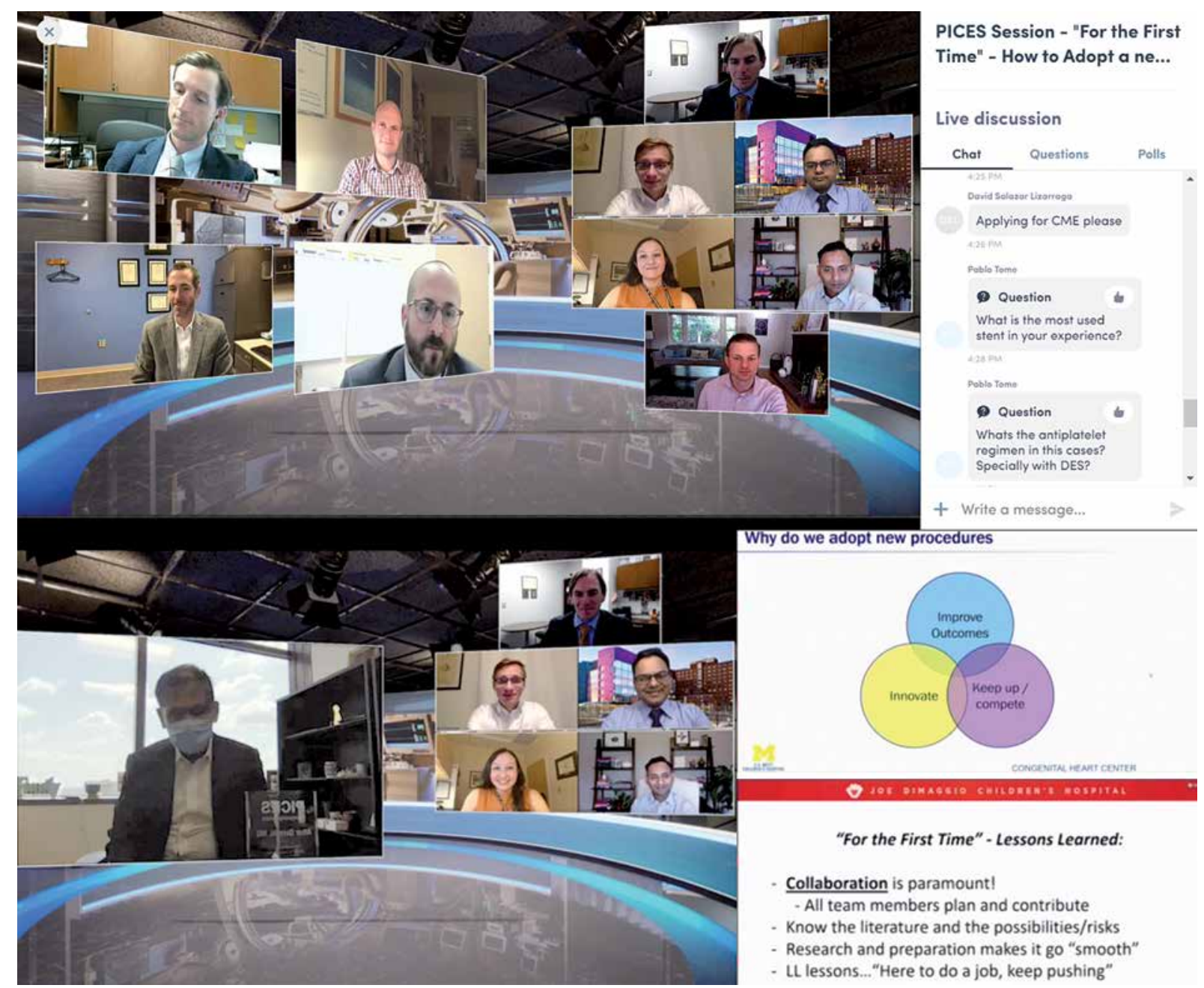

Figure 2. The Paediatric and congenital Interventional Cardiology Early-career Society (PICES) session at the Paediatric and Adult Interventional Cardiac Symposium (PICS-AICS) 

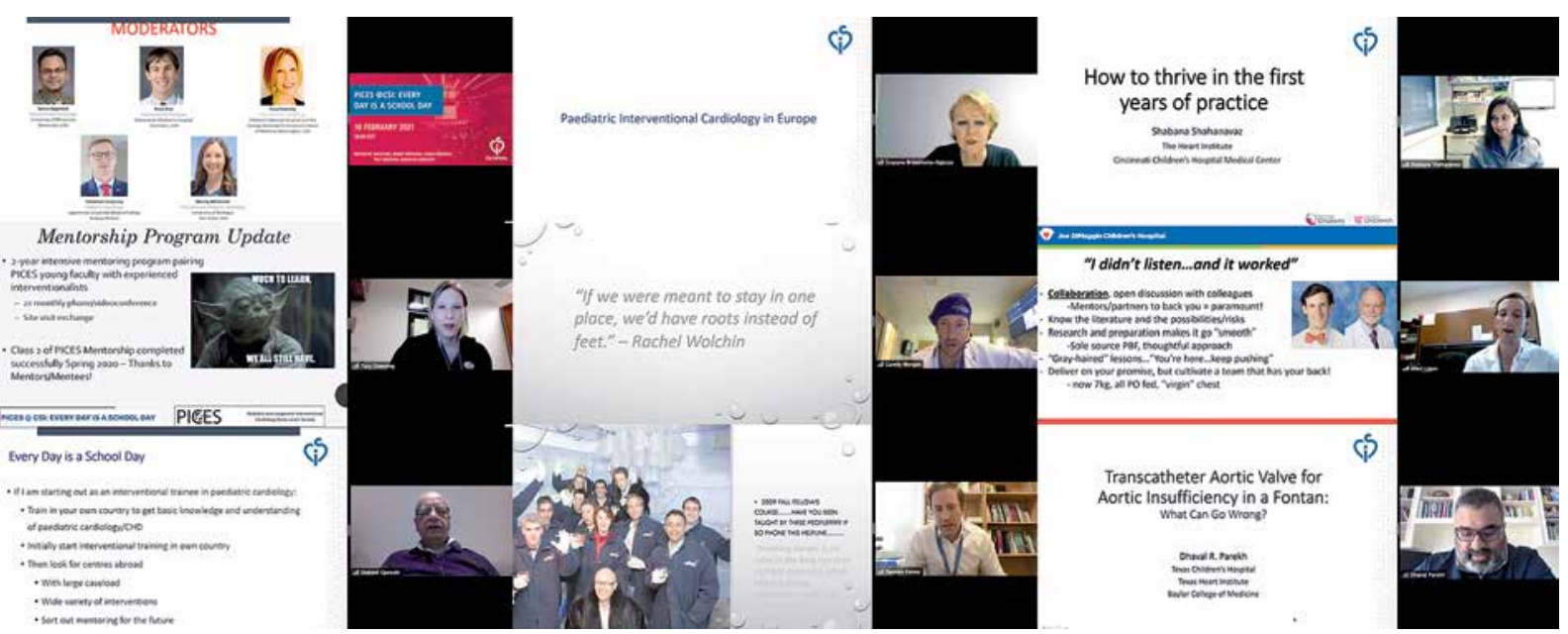

Figure 3. The Paediatric and congenital Interventional Cardiology Early-career Society (PICES) session at the Congenital and Structural Interventions (CSI) webinar

2020) started with our annual PICES Mentorship Award, which was given to the accomplished and humble Dr. Athar Qureshi. See below for quotes from some of his peers:

- "Sincere, passionate, hard-working, supportive, dedicated to training, technically second to none, patients love him, trainees adore him, loved by all and has shaped so many careers across the world...." - Varun Aggarwal, MD.

- "He is the doctor, teacher, mentor, and sponsor I wish everyone could have... lucky to have trained under him" - Kriti Puri, MD.

- "One of the most influential people to shape my training...absolutely dedicated to his patients, has second-to-none technical ability... feel so privileged to have had the opportunity to be trained by him..."

- Chris Broda, MD.

The PICES Mentorship Award was established in 2018 and is presented to a senior interventional cardiologist who has helped develop and support PICES members. Previous recipients include Ziyad Hijazi, MD (2018) and Lee Benson, MD (2019).

The academic portion of the PICES breakout session was titled "For the First Time - How to Adopt a New Procedure in the Lab" and consisted of 3 case presentations from PICES members and an invited lecture from previous PICES Vice President of Research, Jeff Zampi, MD (C.S. Mott Children's Hospital). The session focused on how interventionalists with various levels of experience plan novel procedures and institute new standards of care in the catheterization lab.

The breakout session included case presentations by Allen Ligon, MD (Joe DiMaggio Children's Hospital) on mitral valve interventions, by Daniel McClennan, MBBS (University of lowa) on performing catheterizations through an ECMO circuit, and by Nicholas Hayes, BSc, MBChB, MRCPCH, (Southampton General Hospital, Southampton, UK) on a hybrid tricuspid valve-in-valve procedure. The session concluded with a presentation titled "Starting a PDA Stenting Program (despite excellent surgical outcomes): From Our First Unsuccessful Case to Today" by Jeff Zampi, MD (C.S. Mott Children's). The audience heard an incredibly diverse group of topics and kept the chat box full of thought-provoking questions.

\section{PICES@CSI}

The 2020 CSI Frankfurt meeting was converted to a series of webinars. "Every Day is a School Day" was the title of the PICES session, which focused on the challenges of interventional training and early career development (Figure 3). Various issues related to the interventional fellowship, challenges, and benefits of training in various healthcare systems, including tips for building an independent interventional career, were discussed.

At the beginning of the session Sebastian Goreczny, $M D, P h D$ presented recorded interviews with recognized trainers from around the globe on what makes a good candidate for interventional cardiology training. Grazyna Brzezinska-Rajszysz, MD, PhD (Children's Memorial Hospital, Poland) and Shakeel Qureshi, MD (Evelina London Children's Hospital, UK) then presented training systems in interventional cardiology across Europe and shared valuable tips on how to make the best of an interventional fellowship. Subsequently 2 former PICES executive committee members and globally recognized interventionalists, Gareth Morgan, MD (Colorado Children's Hospital, USA) and Damien Kenny, MD (Our Ladies Children's Hospital, Ireland), used an innovative interview format to discuss the benefits and challenges of perusing interventional training on both sides of the Atlantic. Shabana Shahanavaz, MD (Heart Institute at Cincinnati Children's Hospital Medical Center, USA), another outstanding former PICES member, shared thoughts on how to thrive in the first years of practice. Finally, 2 current PICES members: 
Allen Ligon, MD (Joe DiMaggio Children's Hospital, USA) and Dhaval Parekh, MD (Texas Children's Hospital, USA) presented 2 distinct cases with memorable lessons: percutaneous duct stenting in a duct-dependent pulmonary circulation and aortic valve implantation for aortic insufficiency in a Fontan patient, respectively. Subsequent discussion including questions for the audience extended the webinar beyond the scheduled time. This session was very well attended and is available in an on-demand format (https://www.csi-congress.org/pices-csi-every-dayschool-day).

\section{Future perspectives}

The academic medical community had to adapt to 2020. As we move into a more optimistic 2021, there is hope for the resurgence of in-person conferences, which will facilitate productivity and networking. PICES will continue to support its members through existing programs and lessons learned over the previous year. In 2021, PICES will regularly schedule virtual "fireside chats" to help support the mission of providing support and community to its members. These sessions are intended to be a forum for informal discussion of cases to help plan and solicit opinions on complex upcoming cases, or to discuss challenges and seek alternative approaches to past cases. The PICES website is undergoing significant updates to improve both internal and external communication (https://scai.org/pediatric-interventional-cardiology-early-career-society). These additions should strengthen the PICES community and collaboration among its members.

\section{Conflict of interest}

The authors declare no conflict of interest.

\section{References}

1. Gordon BM, Goldstein BH. Development of an early career society for pediatric and congenital interventional cardiologists: the PICES story. Catheter Cardiovasc Interv 2016; 88: 253-4.

2. Driggin E, Madhavan MV, Bikdeli B, et al. Cardiovascular Considerations for Patients, Health Care Workers, and Health Systems During the COVID-19 Pandemic. J Am Coll Cardiol 2020; 75: 2352-71.

3. Góreczny S, Trucco S, Batlivala SP, et al. Highlights from the Paediatric and Congenital Interventional Cardiology Early-Career Society (PICES) 2019 Activities. Adv Interv Cardiol 2020; 16: 15-8.

4. Seckeler MD, Gordon BM, Williams DA, Goldstein BH. Use of smart technology for remote consultation in the pediatric cardiac catheterization laboratory. Congenit Heart Dis 2015; 10: E288-94.

5. Danon S, Gray RG, Crystal MA, et al. Expansion characteristics of stents used in congenital heart disease: serial dilation offers improved expansion potential compared to direct dilation: results from a Pediatric Interventional Cardiology Early Career Society (PICES) Investigation. Congen Heart Dis 2016; 11: 741-50.

6. Gray RG, Menon SC, Johnson JT, et al. Acute and midterm results following perventricular device closure of muscular ventricular septal defects: a multicenter PICES investigation. Catheter Cardiovasc Interv 2017; 90: 281-9.

7. Aldoss $\mathrm{O}$, Goldstein BH, Danon S, et al. Acute and mid-term outcomes of stent implantation for recurrent coarctation of the aorta between the Norwood operation and fontan completion: a multi-center Pediatric Interventional Cardiology Early Career Society Investigation. Catheter Cardiovasc Interv 2017; 90: 972-9.

8. Crystal MA, Morgan GJ, Danon S, et al. Serial versus direct dilation of small diameter stents results in a more predictable and complete intentional transcatheter stent fracture: a PICES Bench Testing Study. Pediatr Cardiol 2018; 39: 120-8.

9. Taggart NW, Gordon BM, Morgan GJ, Goldstein BH. Variation in anticoagulation practices in the congenital cardiac catheterization lab: results of a multinational PICES survey. Pediatr Cardiol 2019; 40: 53-60.

10. Zampi JD, Alghanem F, Yu S, et al. Relationship between time to left atrial decompression and outcomes in patients receiving venoarterial extracorporeal membrane oxygenation support: a multicenter pediatric interventional cardiology early-career society study. Pediatr Crit Care Med 2019; 20: 728-36.

11. Hiremath G, Morgan G, Kenny D, et al. Balloon expandable covered stents as primary therapy for hemodynamically stable traumatic aortic injuries in children. Catheter Cardiovasc Interv 2020; 95: 477-83.

12. Shahanavaz S, Aldoss O, Carr K, et al. Acute and medium term results of balloon expandable stent placement in the transverse arch-a multicenter pediatric interventional cardiology early career society study. Catheter Cardiovasc Interv 2020; 96: 1277-86. 